\title{
Modeling transmission dynamics of severe acute respiratory syndrome coronavirus 2 in São Paulo, Brazil
}

\author{
Pedro Alexandre da Cruz ${ }^{[1]}$, Leandra Cristina Crema-Cruz ${ }^{[1]}$ \\ and Fabrício Souza Campos ${ }^{[1],[2]}$
}

[1]. Universidade Federal do Tocantins, Colegiado de Ciências Exatas e Biotecnológicas, Campus de Gurupi, Gurupi, TO, Brasil. [2]. Universidade Federal do Tocantins, Laboratório de Bioinformática e Biotecnologia, Campus de Gurupi, Gurupi, TO, Brasil

\begin{abstract}
Introduction: Severe acute respiratory syndrome coronavirus 2 has been transmitted to more than 200 countries, with 92.5 million cases and 1,981,678 deaths. Methods: This study applied a mathematical model to estimate the increase in the number of cases in São Paulo state, Brazil during four epidemic periods and the subsequent 300 days. We used different types of dynamic transmission models to measure the effects of social distancing interventions, based on local contact patterns. Specifically, we used a model that incorporated multiple transmission pathways and an environmental class that represented the pathogen concentration in the environmental reservoir and also considered the time that an individual may sustain a latent infection before becoming actively infectious. Thus, this model allowed us to show how the individual quarantine and active monitoring of contacts can influence the model parameters and change the rate of exposure of susceptible individuals to those who are infected. Results: The estimated basic reproductive number, $R_{o}$, was 3.59 (95\% confidence interval [CI]: 3.48 - 3.72). The mathematical model data prediction coincided with the real data mainly when the social distancing measures were respected. However, a lack of social distancing measures caused a significant increase in the number of infected individuals. Thus, if social distancing measures are not respected, we estimated a difference of at least 100,000 cases over the next 300 days. Conclusions: Although the predictive capacity of this model was limited by the accuracy of the available data, our results showed that social distancing is currently the best non-pharmacological measure.
\end{abstract}

Keywords: SARS-CoV-2. COVID-19. SEIR model transmission dynamics. Mathematical modelling.

\section{INTRODUCTION}

Severe acute respiratory syndrome coronavirus 2 (SARSCoV-2) belongs to the family Coronaviridae ${ }^{1,2}$. SARS-CoV-2 has been transmitted to more than 200 countries with 96.0 million cases and 2,049,232 deaths worldwide ${ }^{3,4}$. The coronavirus disease (COVID-19) has devastated health, economic, and social infrastructures worldwide and is considered the largest pandemic crisis of the 21st century. SARS-CoV-2 emerged in Wuhan, China, in December 2019. The local epidemic rapidly spread to multiple countries, with consequent challenges for surveillance and control ${ }^{5}$. The first case of SARS-CoV-2 infection in Brazil was confirmed

\footnotetext{
Corresponding author: Prof. Dr. Pedro Alexandre da Cruz. e-mail: pedrocruz@uft.edu.br

(iD) https://orcid.org/0000-0002-7208-8545

Received 13 August 2020

Accepted 05 January 2021
}

on February 26, 2020, in São Paulo (SP), the 8th largest city in the world, with 12 million inhabitants ${ }^{6}$.

No treatment is available to date, and vaccines are not expected to be sufficiently widely available to control the SARS-CoV-2 pandemic within the coming year. The only current approaches to reduce the number of new cases and the transmission rate during this pandemic are those of classical epidemic control, including case isolation, contact tracing and quarantine, physical distancing, and hygiene measures ${ }^{7}$. Additionally, knowledge of the propagation pattern of COVID-19 and the prediction of the time evolution is of great importance to save lives and reduce the social and economic consequences of the disease ${ }^{8}$. These data can be incorporated by mathematical models to understand how SARS-CoV-2 spreads within a population.

Since SARS-CoV-2 transmission started in Wuhan, China, mathematical modeling has been at the forefront of shaping the 
decisions regarding non-pharmaceutical interventions to confine its spread worldwide ${ }^{9,10}$. The viral spread can be determined by observing the period of incubation (the period during which an infected individual shows nonspecific or early symptoms during the prodromal phase, before classical clinical symptoms) and can be represented by the susceptible exposed infected recovered (SEIR) model to evaluate how social measures of isolation and quarantine can alter mortality rates and the number of cases of infected individuals over time. Another factor to consider is the basic reproduction number $\left(R_{0}\right)$, used to measure the potential transmission of a disease ${ }^{11}$.

The SEIR-A mathematical model proposed by Yang and Wang ${ }^{12}$ has been used to study the dynamic spread of SARS-CoV-2 in Wuhan, China. We adapted this model and applied it in SP state, Brazil. Parameters such as SARS-CoV-2 surface stability and environment-human and human-human routes were considered to demonstrate how quarantine and social distancing can help in controlling the pandemic. Likewise, the lack of these nonpharmaceutical interventions can increase the spread of SARS$\mathrm{CoV}-2$ and prolong the pandemic period in Brazil.

\section{METHODS}

\section{Mathematical Modeling}

The mathematical model to describe the SARS-CoV-2 transmission in SP state divided the entire population into five classes: susceptible $(S)$, exposed $(E)$, infected $(I)$, recovered $(R)$, and environmental reservoir $(A)$ class. The infected and exposed populations (individuals in the incubation period) can infect the susceptible population. Recovered individuals were those who were cured or who died of COVID-19. Finally, class A represented the indirect, environment-to-human transmission rate. SARS-CoV-2 spread among these classes and its circulation are represented in Figure 1.

Membership in the classes changes over time and one can conceptualize the time course of a pandemic as a movement of hosts among classes. Thus, the diagram shown in Figure 1 leads to the following system of ordinary differential $(d)$ equations. Each set of dependent variables counts individuals in each of the groups, each as a function of time $(t)$ :

$$
\begin{aligned}
& \frac{d S}{d t}=\Delta-T_{E}(E) S E-T_{I}(I) S I-T_{A}(A) S A-\mu S \\
& \frac{d E}{d t}=T_{E}(E) S E+T_{I}(I) S I+T_{A}(A) S A-(\alpha+\mu) S \\
& \frac{d l}{d t}=\alpha E-\left(m_{D}+\gamma+\mu\right) I
\end{aligned}
$$

$$
\begin{gathered}
\frac{d R}{d t}=\gamma I-\mu R \\
\frac{d A}{d t}=\theta_{1} E+\theta_{2} I-\sigma A,
\end{gathered}
$$

where $\Delta$ is the birth rate of the local population; $T_{E 0}$ is constant transmission between susceptible and exposed individuals $[E T(E) S E] ; T_{A 0}$ is constant transmission between susceptible and infected individuals $\left[T(I) S I ; T_{A 0}\right.$ is constant transmission between susceptible and environmental reservoir $[T(A) S A]$; $\mu$ is natural death rate; $\alpha$ is the incubation period between infection and the onset of disease symptoms; $m_{D}$ is the disease-related death rate; $\gamma$ is the recovery rate for the COVID-19; $\theta_{1}$ is SARS-CoV-2 shedding rate by exposed individuals; $\theta_{2}$ is the rate of SARS-CoV-2 shedding by infected individuals; and $\sigma$ is the rate of SARS-CoV-2 removal from the environment.

The functions $T_{E}(E)$ and $T_{I}(I)$ represent human-to-human transmission rates between exposed and susceptible and between infected and susceptible individuals, respectively, and require adjustment for the transmission coefficient (c), which in this study was given by:

$T_{E}(E)=\frac{T_{E 0}}{1+c E}$ and $T_{I}(I)=\frac{T_{I 0}}{1+c I}$

where $T_{E 0}$ and $T_{I 0}$ express the maximum transmission rates. The function $T_{A}(A)$ represents the environmental-to-human transmission rate and is given by:

$$
T_{A}(A)=\frac{T_{A 0}}{1+c A}
$$

The basic reproduction number $R_{0}$ is defined as the expected number of secondary cases produced by a single (typical) infection in a completely susceptible population ${ }^{13}$. The model used in this study defined $R_{0}$ as:

$R_{0}=\frac{T_{E}(0) S_{0}}{\alpha+\mu}+\frac{\alpha T_{I}(0) S_{0}}{\omega_{1}(\alpha+\mu)}+\frac{\left(\omega_{1} \theta_{1}+\alpha \theta_{2}\right) T_{A}(0) S_{0}}{\sigma \omega_{1}(\alpha+\mu)}=R_{1}+R_{2}+R_{3}$

where, $S_{0}$ is the initial percentage of the susceptible population 


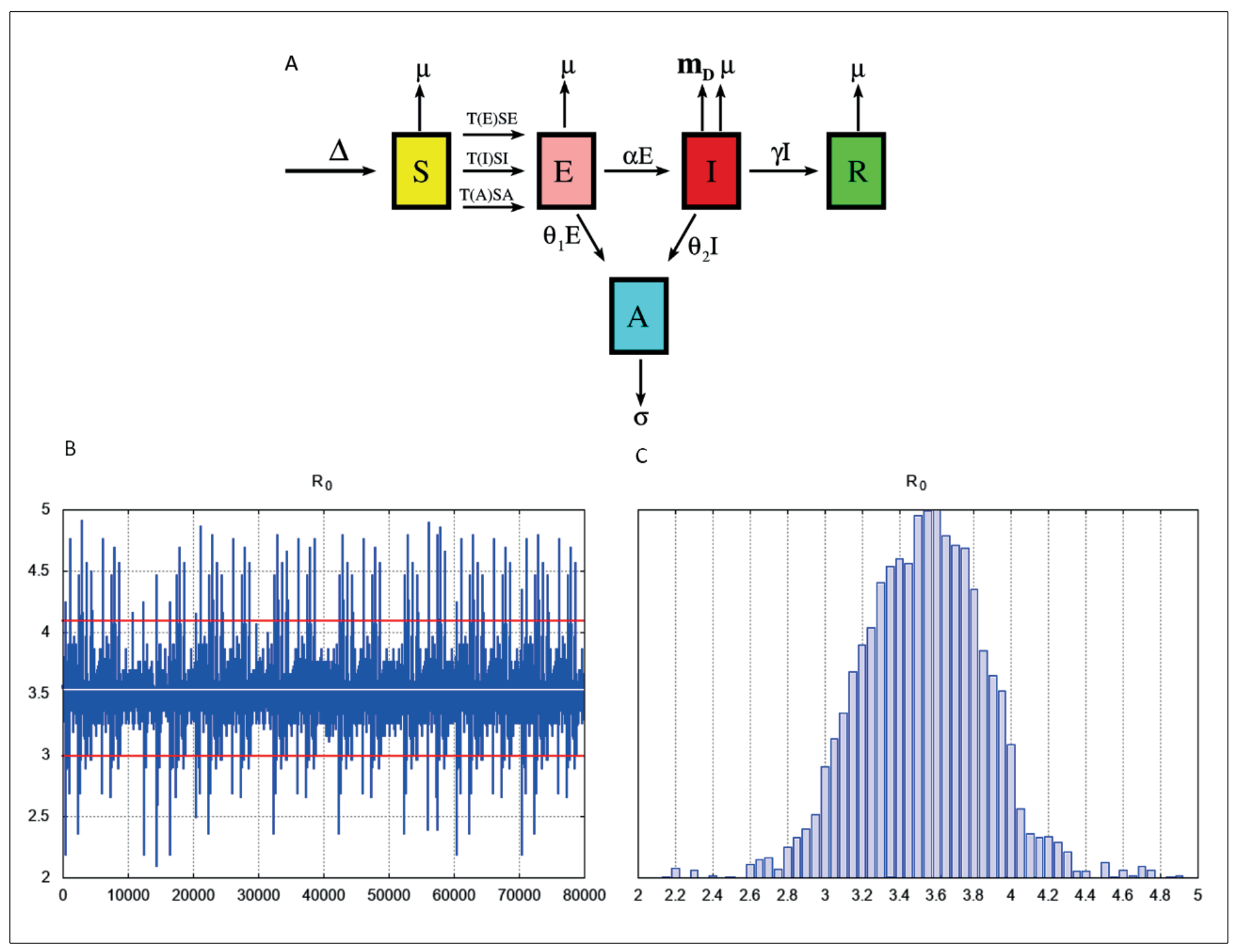

FIGURE 1 (A): Diagram of the SEIR-A model applied in the study to simulate SARS-CoV-2 spread. Each class is represented by its acronym: the susceptible population $(S)$ is exposed to infection by direct and environmental transmission. In the exposed state $(E)$, the population becomes infected $(I)$. Infected individuals either die because of COVID-19 or recover $(R)$. The exposed and infected populations spread the virus in environments $(A)$ that can infect susceptible individuals. $\Delta$ : birth rate of the local population; $\mu$ : natural death rate; $T(E) S E$ : constant transmission between susceptible and exposed individuals; $T(I) S I$ : constant transmission between susceptible and infected individuals; $T(A) S A$ : constant transmission between susceptible individuals and the environmental reservoir; $\theta_{1}$ : rate of SARSCoV-2 shedding by exposed individuals; $\alpha$ : incubation period between infection and the onset of disease symptoms; $\sigma$ : rate of SARS-CoV-2 removal from the environment; $m_{D}$ : disease-related death rate; $\theta_{2}$ : rate of SARS-CoV-2 shedding by infected individuals; $\gamma$ : rate of COVID-19 recovery. (B): Trace plot output of $R_{0}$. (C): Histogram generated by the MCMC method for parameter $R_{0}$.

and $\omega_{1}$ is the sum of $m_{D}, \alpha$ and $\mu$ parameters. Thus, $R_{0}=1$ is a threshold parameter to quantify SARS-CoV-2 spread by estimating the average number of secondary infections in a wholly susceptible population. If $R_{0}<1$, the number of infected individuals decreases over time as SARS-CoV-2 is contained. However, if, the number of infected individuals increases and SARS-CoV-2 persists. The term $R_{1}$ measures the contribution from exposed to susceptible individuals' transmission, while $R_{2}$ measures the contribution from infected to susceptible individuals' transmission. The third term, $R_{3}$, represents the contribution from the environmental-to-human transmission route. These three transmission modes collectively shape the overall infection risk for the SARS-CoV-2 pandemic.

\section{RESULTS}

\section{Parameter estimation and model fitting}

The numerical validation and computational simulations of the mathematical model proposed by the system of equations (2.1) used cumulative reported data from the COVID-19 daily bulletin from the SP city Health Department that has statewide data ${ }^{14}$. The data were based on confirmed testing between February 25 and July 05 , 2020, with 320,179 confirmed infections.

The mathematical model proposed by the system of equations (2.1) was implemented in the mathematical software Octave and 
numerical simulations were performed for an epidemic period between February 25 and July 05, 2020. The estimated population for SP state is over 45 million $^{6}$ and the state was placed under quarantine by the current governor on March 24, 2020. In the epidemic period, our simulations assumed that only a relatively "small" number of people have traveled to SP state; thus, the inflow rate $(\Delta)$ of the model is based only on the number of newborns in the state. Spencer et al. ${ }^{15}$ reported an average recovery period of approximately 15 days; hence, we defined the recovery rate from COVID-19 as $\gamma=1 / 15$ per day. The incubation period of the infection varied between 2 and 14 days, with an average of 5-7; therefore, $\sigma=1 / 15$. Kampf et al. ${ }^{16}$ reported that some members of the Coronaviridae family can remain infectious in the environment from 2 hours up to 5-9 days. We considered several values for the $\sigma$ parameter; namely, $0<=\sigma<=1$, depending on the date of the computer simulation. The transmission rate $\left(T_{E 0}\right.$ and $\left.T_{I 0}\right)$ values were estimated as described by Tang et al. ${ }^{17}$. Additionally, $\theta_{1}$ and $\theta_{2}$ were estimated using a Markov chain Monte Carlo (MCMC) method in our computer simulation (the MCMC method is described in Supplementary Material 1). On March 24, a strict policy of social distancing was implemented, with medical care offered to confirmed cases; thus, SARS-CoV-2 spread by infected individuals to the environment was considered low. Therefore, between March 24 and April 24, we considered $\theta_{2}=0$ and $\theta_{1}>0$.

The present study also considered the presence of SARS-CoV-2 in the environment. For this, three parameters were determined: the adjustment coefficient $(c)$, the rate $\left(\theta_{1}\right)$, and environment-tohuman constant transmission $\left(T_{A 0}\right)$. To estimate the value of $\theta_{1}$, we applied MCMC methods based on the adaptive combination Delayed rejection and Adaptive Metropolis (DRAM) algorithm ${ }^{18,19}$ to the system (2.1) (Supplementary Material 1). We sampled from 80,000 MCMC iterations and discarded the first 10,000 samples as a burn-in period. Based on these 70,000 samples, the point estimates and $95 \%$ confidence intervals (CIs) for those parameters were calculated. Based on the fitted model, the estimated $R_{0}$ was 3.59 (95\% CI: $3.48-3.72$ ), which meant that each infected person could infect an average of 3.59 people during the infection period. Lastly, $\theta_{1}, T_{A 0}$ and $c$ values and $95 \%$ CIs were determined for the four epidemic periods analyzed and were similar to the $R_{0}$ parameter $^{20,21}$. The first conditions for the five classes of the differential equation system and parameter values used in the computational model for the four different simulation periods are shown in Table 1. Using the estimated parameter values, we assessed the fit between the model solution and real data, as shown in Figure 2.

TABLE 1: Initial conditions for the five classes of differential equation system and parameter values used in the computational model.

\begin{tabular}{|c|c|c|c|c|c|c|c|c|c|}
\hline Parameters & First period & Std & $\begin{array}{l}\text { Second } \\
\text { period }\end{array}$ & Std & Third period & Std & Fourth period & Std & Source \\
\hline$T_{\mathrm{A} 0}$ & $4.04 \times 10^{-10}$ & $4.41 \times 10^{-11}$ & $4.15 \times 10^{-10}$ & $4.57 \times 10^{-11}$ & $1.03 \times 10^{-11}$ & $5.44 \times 10^{-12}$ & $1.12 \times 10^{-11}$ & $6.15 \times 10^{-12}$ & This study \\
\hline$c$ & $4.03 \times 10^{-5}$ & $6.17 \times 10^{-6}$ & $4.93 \times 10^{-5}$ & $6.93 \times 10^{-6}$ & $3.71 \times 10^{-6}$ & $9.81 \times 10^{-7}$ & $1.27 \times 10^{-6}$ & $7.38 \times 10^{-7}$ & This study \\
\hline$\theta_{1}$ & 2.376 & 0.426 & 3.786 & 0.535 & 2.135 & 0.394 & 4.052 & 0.547 & This study \\
\hline$S(0)$ & $45,919,049$ & --- & $45,907,329$ & ---- & $45,854,629$ & ---- & $45,511,907$ & ----- & 22 \\
\hline$E(0)$ & 1 & ---- & 800 & ---- & 15,000 & ----- & 100,000 & ---- & 14 \\
\hline$I(0)$ & 1 & ---- & 820 & ---- & 18,420 & ----- & 107,142 & ---- & 14 \\
\hline$R(0)$ & 0 & ---- & 100 & ---- & 1,000 & ----- & 100,000 & ---- & 14 \\
\hline$A(0)$ & 6 & ---- & 10,000 & ---- & 30,000 & ---- & 100,000 & ---- & 14 \\
\hline$T_{\mathrm{E} 0}$ & $6.32 \times 10^{-9}$ & $7.11 \times 10^{-10}$ & $6.02 \times 10^{-9}$ & $6.83 \times 10^{-10}$ & $5.02 \times 10^{-9}$ & $4.14 \times 10^{-10}$ & $4.52 \times 10^{-9}$ & $3.38 \times 10^{-10}$ & 17 \\
\hline$T_{I 0}$ & $3.32 \times 10^{-9}$ & $7.92 \times 10^{-10}$ & $1.22 \times 10^{-9}$ & $6.67 \times 10^{-10}$ & $1.01 \times 10^{-9}$ & $6.04 \times 10^{-10}$ & $7.61 \times 10^{-10}$ & $4.74 \times 10^{-11}$ & 17 \\
\hline$\theta_{2}$ & 1.037 & 0.373 & 0.00 & ---- & 1.247 & 0.389 & 0.863 & 0.291 & This study \\
\hline$\Delta$ & $1,659.26$ & ----- & $1,659.26$ & ---- & $1,659.26$ & ----- & $1,659.26$ & ----- & 22 \\
\hline$m_{D}$ & $0.0372 /$ day & ---- & $0.045 /$ day & ---- & $0.05 /$ day & ---- & $0.05 /$ day & ---- & 14 \\
\hline$\mu$ & $3.5 \times 10^{-5} /$ day & ----- & $3.5 \times 10^{-5} /$ day & ---- & $3.5 \times 10^{-5} /$ day & ---- & $3.5 \times 10^{-5} /$ day & ----- & 22 \\
\hline$\alpha$ & 5 days & ---- & 5 days & --- & 5 days & ---- & 5 days & ---- & 15 \\
\hline$\gamma$ & 1/15/day & ---- & 1/15/day & ---- & 1/15/day & ---- & 1/15/day & ---- & 15 \\
\hline$\sigma$ & $0.2 /$ day & ----- & 1/day & ---- & $0.2 /$ day & ---- & $0.2 /$ day & ---- & 23 \\
\hline
\end{tabular}

$T_{A 0}$ : constant transmission between susceptible individuals and the environmental reservoir; $c$ : transmission coefficient; $\theta_{1}:$ rate of SARS-CoV-2 shedding by exposed individuals; $S(0)$ : susceptible individuals; $E(0)$ : exposed individuals; $I(0)$ : infected individuals; $R(0)$ : recovered individuals; $A(0)$ : environmental reservoir; $T_{\mathrm{E}}$ : constant transmission between susceptible and exposed individuals; $T_{\mathrm{I}}$ : constant transmission between susceptible and infected individuals; $\theta_{2}$ : rate of SARS-CoV-2 shedding by infected individuals; $\Delta$ : birth rate of the local population; $m_{D}$ : disease-related death rate; $\mu$ : natural death rate; $\alpha$ : incubation period between infection and the onset of disease symptoms; $\gamma$ : recovery rate from COVID-19; $\sigma:$ rate of SARS-CoV-2 removal from the environment 


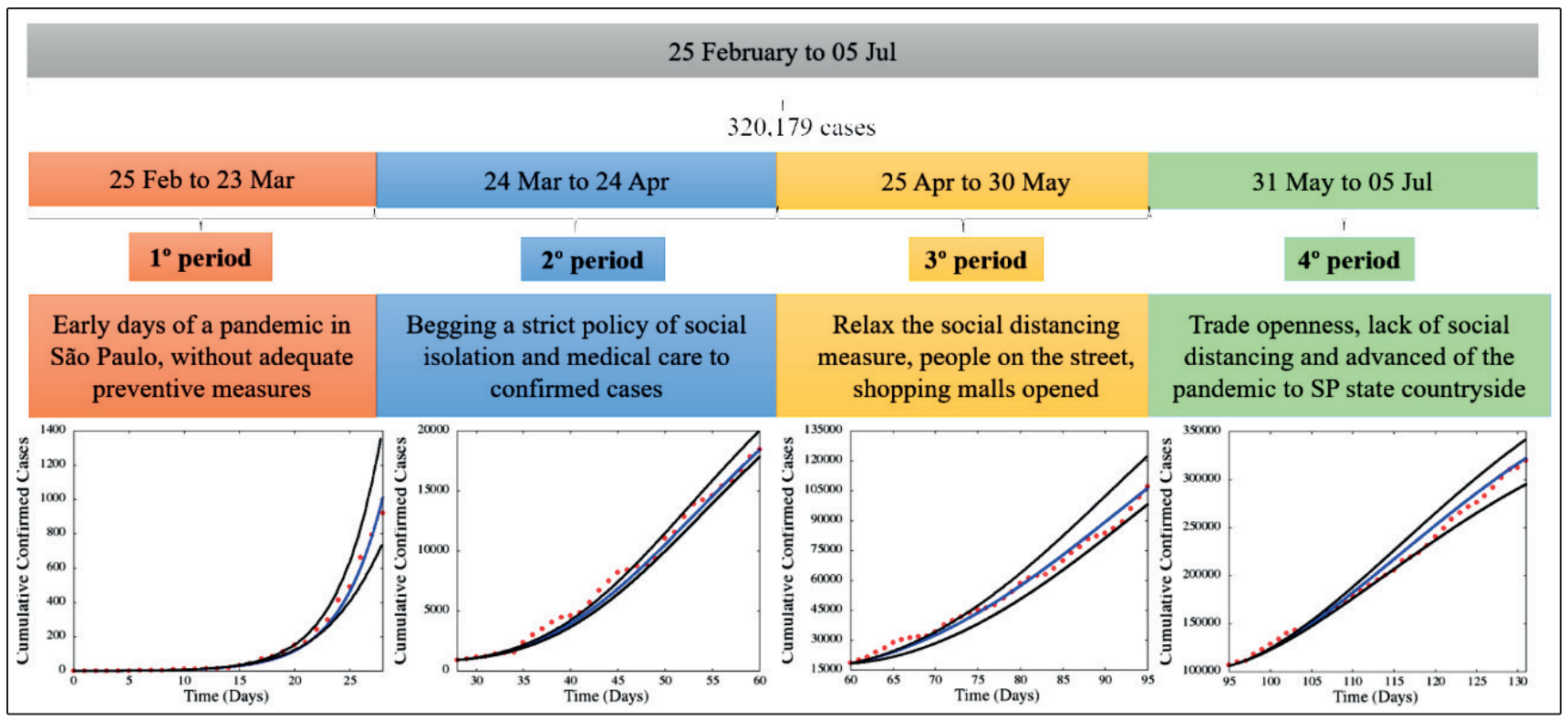

FIGURE 2: Cumulative confirmed cases in four different periods. In the graphs at the bottom of the figure, the solid blue line denotes the result of the computer simulation, the red balls denote the reported cases of COVID-19, and the solid black lines represent the lower and upper bounds of the $95 \%$ Cl for all 10,000 simulations.

\section{Numerical results}

To illustrate the estimated $R_{0}$ before the quarantine (the first period), Figure $1 \mathbf{b}$ shows a trace plot of the MCMC output using 80,000 MCMC samples. The histograms of $R_{0}$ values generated by the MCMC method are shown in Figure 1c.

The estimated $R_{0}$ was 3.59 before the quarantine (first period). For the second, third, and fourth periods, we instead estimated the effective reproductive number $\left(R_{\mathrm{t}}\right)$. The estimated $R_{\mathrm{t}}$ values were 1.972 (95\% CI: 1.535 - 2.427), 1.753 (95\% CI: 1.253 - 2.239) and 1.558 (95\% CI: $0.973-1.879)$ in the second, third, and fourth periods, respectively. The numbers of cumulative confirmed cases for the four epidemic periods of COVID-19 in SP state versus the adjustment curves are shown in Figure 2. We observed a good fit between the model solution and real data with $95 \%$ CIs for all 10,000 simulations. The good agreement between solutions validated our results.

We used a computational mathematical model to determine the trend in the numbers of cumulative cases of infected and exposed individuals (Figure 3). The numerical simulation to the first period showed that the infection level increased up to 90-100 days (Figure 3A), peaking at around 124,000 infected individuals on June 4, 2020. In the second period, with a policy of maintaining social distancing, the numerical simulation showed that the infection level increased up to 65-70 days, peaking at approximately 36,000 infected individuals on June 2, 2020 (Figure 3B). During the third period, with the relaxing of social distancing measures, the infection level increased up to 80-90 days, peaking at approximately 352,500 infected individuals on July 25, 2020 (Figure 3C). Finally, in the fourth period, with trade openness, lack of social distancing, and advancing of the pandemic to the SP countryside, the infection level increased up to 60-70 days, peaking at approximately 718,610 infected individuals on July 05, 2020 (Figure 3D).

\section{Variations in $\theta_{2}$}

The $\theta_{2}$ value increased when there was a reduction in social distancing, reflecting the number of individuals infected by SARSCoV-2 (Table 2). Variations in the numbers of confirmed cases for different $\theta_{2}$ values are shown in Figure 3E. When $\theta_{2}=0$, the contribution of infected individuals, like the SARS-CoV-2 environmental reservoir, is low. The predicted number of cases on March 23 was 779, a value below the actual number of confirmed cases (860). When $\theta_{2}=1$, about 18,265 cases were predicted for April 24, a number that differed slightly from the actual number of confirmed cases $(17,826)$. However, when $\theta_{2}=10$, the model predicted 4,830 cases on March 23, different from the actual number of confirmed cases (860).

\section{Variations in $\sigma$}

The $\sigma$ parameter in the SEIR-A model indicates the rate of SARSCoV-2 removal from the environment. Variations in the confirmed numbers of cases for different $\sigma$ values are shown in Figure 3. The effects of SARS-CoV-2 removal rate in the first period, when $\sigma=0.2$ (green line in Figure 3F) suggested that approximately 5 days were required to decrease SARS-CoV-2 in the environment ${ }^{12}$. During this period, the number of cases predicted by our model (890) was consistent with the actual number of confirmed cases (830). A removal rate $(\sigma)$ of 1 suggested that approximately 1 day was required to decrease SARS-CoV-2 circulation in the environment ${ }^{12}$, with 314 predicted infections, a number smaller than the actual number of confirmed cases. In the second period $\left(\theta_{2}=0\right.$ and $\left.\sigma=1\right)$, there were 17,840 predicted infections on April 24 (red line on Figure 3G), very close to the actual number of confirmed cases $(17,826)$. 
TABLE 2: Predictions of confirmed cases for $\sigma=0.2\left(1^{\circ}\right.$ period $)$ and $\sigma=1\left(2^{\circ}\right.$ period) with different values of $\theta_{2}$ parameters.

\begin{tabular}{|c|c|c|c|c|c|c|c|c|c|c|c|c|c|c|}
\hline Date & $25 / 02$ & $01 / 03$ & $06 / 03$ & $12 / 03$ & $17 / 03$ & $20 / 03$ & $23 / 03$ & $25 / 03$ & $30 / 03$ & $05 / 04$ & $11 / 04$ & $16 / 04$ & $20 / 04$ & $24 / 04$ \\
\hline Predicted confirmed cases $\theta_{2}=0$ & 1 & 2 & 7 & 38 & 153 & 349 & 779 & 860 & 1,657 & 4,661 & 8,057 & 11,132 & 14,276 & 17,840 \\
\hline Predicted confirmed cases $\theta_{2}=1$ & 1 & 2 & 8 & 46 & 174 & 411 & 890 & 860 & 1,680 & 4,781 & 8,251 & 11,391 & 14,541 & 18,265 \\
\hline Predicted confirmed cases $\theta_{2}=5$ & 1 & 2 & 12 & 92 & 473 & 1,171 & 2,617 & 862 & 1,757 & 5,142 & 8,813 & 12,057 & 15,255 & 18,890 \\
\hline Predicted confirmed cases $\theta_{2}=10$ & 1 & 3 & 18 & 174 & 981 & 2,347 & 4,830 & 863 & 1,827 & 5,437 & 9,245 & 12,550 & 15,765 & 19,366 \\
\hline Real data of confirmed cases & 1 & 1 & 10 & 42 & 164 & 413 & 860 & 862 & 1,537 & 4,620 & 8,216 & 11,043 & 14,267 & 17,826 \\
\hline
\end{tabular}

б: rate of SARS-CoV-2 removal from the environment; ${ }^{\circ}$ period: February 25 to March 23, 2020; ${ }^{\circ}$ period: March 24 to April 24,$2020 ; \theta_{2}$ : rate of SARS-CoV-2 shedding by infected individuals.
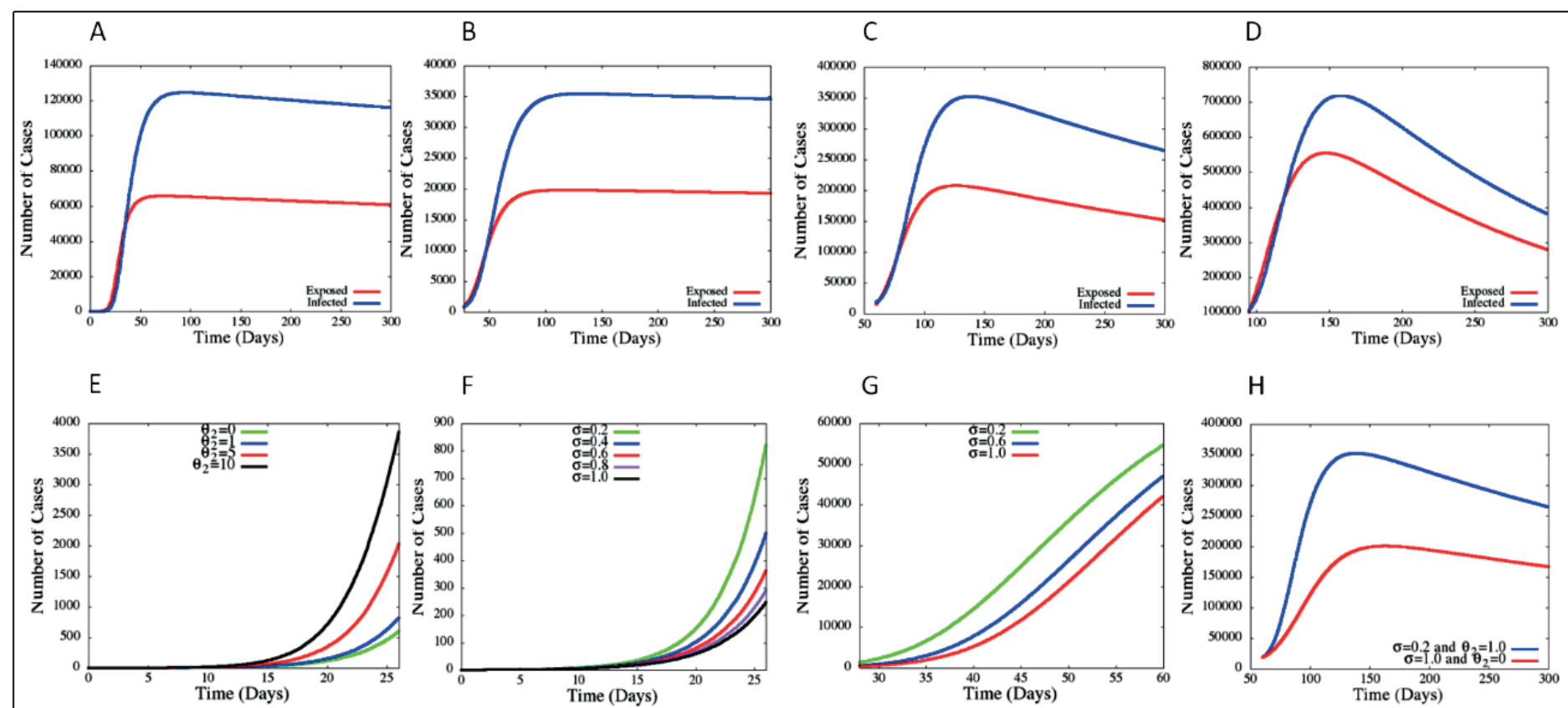

$$
\text { F }
$$

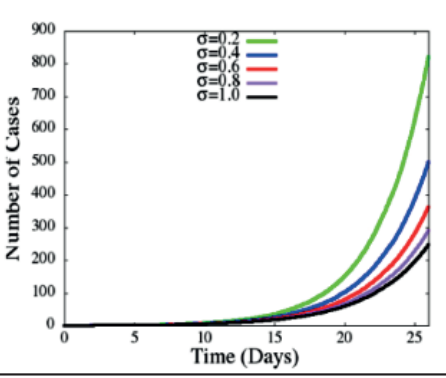

G

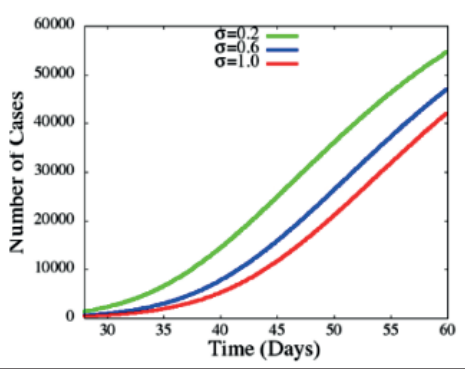

$\mathrm{H}$

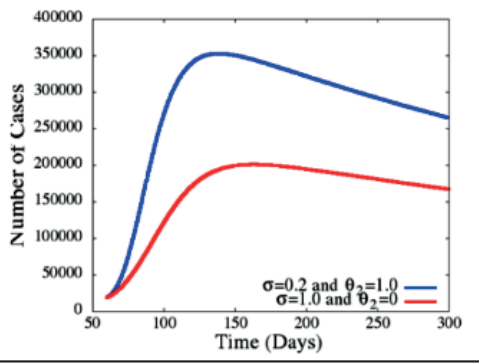

FIGURE 3: Results of numerical simulations to predict the cumulative number of SARS-CoV-2 infected and exposed individuals in SP state during four different time periods (A to D), as well as the effects of the rate of SARS-CoV-2 removal from the environment in SP state among confirmed cases of infection. (E): First period and $\theta_{2}=1$. (F): Second period and $\theta_{2}=0$. (G): Match effects of the policy of social distancing $\left(\theta_{2}\right)$ and the removal rate of SARS-CoV-2 $(\sigma)$ from the environment in SP state from confirmed cases in the first period. (H): Projection of individuals infected between April 25, 2020, and February 19, 2021 (300 days later).

The effects of the social distancing policy (measured by $\theta_{2}$ ) and the rate of SARS-CoV-2 removal from the environment (measured by $\sigma$ ) are shown in Figure 3H (red line) from the time of the initial implementation of the strict social distancing, indicating the projected number of people infected between April 25, 2020, and February 19, 2021 (300 days later). The results of our model showed that maintaining non-therapeutic measures resulted in 170,000 rather than 270,000 cases'.

\section{DISCUSSION}

This study applied an SEIR-A model that considered the potential routes from the reservoir to a person and from person to person of SARS-CoV-2, respectively, to compare the estimated data to the reported data for four epidemic periods of COVID-19 in SP state, Brazil. All scenarios showed agreements between the numerical solutions obtained via the mathematical model and the actual data on the number of confirmed cases. Moreover, the SEIR-A model was also used to predict SARS-CoV-2 spread in SP state for the next 300 days.

The model incorporated multiple transmission pathways as well as an environmental class that represented the pathogen concentration in the environmental reservoir. Here, the term "environmental reservoir" refers to the presence of SARS-CoV-2 in urban areas based on findings reported by Abrahão et al. ${ }^{24}$ regarding the detection of SARS-CoV-2 RNA on public surfaces in a densely populated urban area in Brazil. Using sterile swabs, the authors evaluated 101 samples collected from different surfaces near the hospital and public transportation sites and submitted them for nucleic acid extraction and genomic detection and quantification by one-step quantitative polymerase chain reaction (qPCR). Seventeen (16.8\%) samples collected from bus stations, public squares, and sidewalks tested positive for SARS-CoV-2 RNA, including samples obtained near hospitals. Thus, the study results demonstrated the 
contamination of public surfaces by SARS-CoV-2, especially near hospital areas, highlighting the risk of infection. Additionally, the US Centers for Disease Control and Prevention (CDC) $)^{25}$ also recognizes the risk for individuals to be infected by SARS-CoV-2 by touching a surface or object contaminated with the virus and then touching their mouths, noses, or eyes. While this is not thought to be the main route of viral spread, we are still learning more about how this virus spreads.

To better understand how the virus spreads among people and via objects, Böhmer et al. ${ }^{26}$ studied the transmission of SARS-CoV-2 from patient 0 (a Chinese resident who visited Germany for professional reasons) until the infection of patient 16 . The infection of patient 5 by patient 4 happened in a single encounter during a canteen visit, with the patients sitting back-to-back, when patient 5 borrowed a saltshaker from patient 4 , thus demonstrating the potential for contamination via objects. Thus, the environment acts as a reservoir for SARS-CoV-2 and can lead to the infection of susceptible individuals.

To prevent individual and community transmission, an accurate test for SARS-CoV-2 and appropriate preventive measures are paramount ${ }^{27}$. As the epidemic progresses, all tools available for SARS-CoV-2 diagnosis must be applied. COVID-19 daily bulletin data from SP city Health Department ${ }^{14}$ contains the results of reverse transcription-qPCR (RT-qPCR), rapid tests for antibody and antigen detection, enzyme-linked immunosorbent assay (ELISA) tests, and other types of tests. While RT-qPCR detects active SARS-CoV-2 infection, serological tests based on immunoglobulin $\mathrm{G}(\mathrm{IgG})$ show previous exposure to SARS-CoV-2. These differences impact our estimates, especially the numbers of infected individuals. However, the underreporting of cases and high percentages of asymptomatic and pre-asymptomatic individuals also contribute to the spread of SARS-CoV- $2^{28}$. Thus, the data generated in our study should be used with caution.

The basic reproduction number $R_{0}$ is a powerful quantitative concept used to characterize the contagiousness and transmissibility of SARS-CoV-2 ${ }^{29,30}$. This number reflects how new infections are caused by a single infectious individual in an otherwise completely susceptible population ${ }^{30,31}$. The $R_{0}$ in all scenarios in our simulations was $>1$ (3.59 to 1.558$)$, with greater values observed when no measures had been implemented to prevent virus spread, as occurred in Wuhan, China ${ }^{32} \cdot R_{0}>1$ indicated the highest number of infected people and the consequent persistence of SARS-CoV-2 in SP state.

Comparison of the results obtained in the numerical simulations to real data from the confirmed cases showed that the mathematical modeling satisfactorily predicted the cases that occurred in the first period (February 25 to March 23, 2020) (Table 2). In particular, the predictions on March 20 and 23, 2020 were approximately 411 and 890 cases, nearly identical to the number of confirmed cases on those dates (413 and 860). During the second period, approximately 14,276 and 17,840 cases were predicted for April 20 and 24, 2020 were, respectively, also very close to the actual number of confirmed cases of 14,267 and 17,826. However, the discrepancy observed between the predicted and confirmed cases was directly related to the relaxation of social distancing measures. Because of the greater number of infected people, the virus spread in the environment ${ }^{33}$.
In contrast, the removal of SARS-CoV-2 from the environment decreases the number of confirmed infected cases according to the increase in $\sigma$. Thus, measures like hospitalization or isolation of individuals with positive diagnoses, tracking of new cases, and strict isolation to reduce contact with infected individuals will increase the rate of removal of SARS-CoV-2 from the environment, reflecting a smaller number of cases $(100,000$ fewer cases over the next 300 days). Respiratory infectious diseases, such as those caused by SARS-CoV-2, are spread through a susceptible individual's contact with the virus. These contacts facilitate disease transmission and can be made indirectly through environmental routes or direct person-toperson interactions $\mathrm{s}^{33}$. Thus, measures such as wearing masks, social distancing, isolation of positive cases, and tracking of new cases are essential to mitigating the COVID-19 pandemic in SP state, Brazil and, therefore, must be enforced by the government in the form of law.

We emphasize that the mathematical model has limitations. We used official data from the State Health Secretariats, which releases data after some days of delay. It is important to consider that, in Brazil, people hospitalized or who come to the hospital with flulike signs, and sometimes, contacts of positive patients, are tested for SARS-CoV-2 infection. Thus, the number of cases considered positive may be higher than the reported cases, which does not invalidate our results because the most significant population in this study was patients requiring medical care, who can lead to the collapse of the public health system. Therefore, the results of in study can be used to evaluate the effects of a strict policy of social isolation, preventive measures, and decisions for new strategies to reduce the SARS-CoV-2 pandemic.

In conclusion, we used a mathematical model to show the effects of social distancing on the number of cases of SARSCoV-2 infection during the pandemic in SP state. We showed that the discrepancy observed between the predicted and confirmed numbers of cases was directly related to the relaxation of social distancing measures. Therefore, the duration of social distancing has significantly decreased the number of infected people in SP state. Our model showed that maintaining non-therapeutic measures resulted in 170,000 rather than 270,000 cases at the end of 300 days. Thus, if we do not have a SARS-CoV-2 vaccine, we believe that non-therapeutic measures are the best strategy to combat the disease.

\section{ACKNOWLEDGEMENTS}

We offer our deepest thanks to the Coordination of Health Surveillance in the city of São Paulo for the data made available and updated on the São Paulo City Hall website.

\section{FINANCIAL SUPPORT}

This research was supported by the Federal University of Tocantins, Campus of Gurupi and by the corresponding author's own resources.

\section{CONFLICT OF INTERESTS}

The authors declare that they have no conflict of interests.

\section{REFERENCES}

1. Gorbalenya AE, Baker SC, Baric RS, Groot RJ, Drosten C, Gulyaeva $\mathrm{AA}$, et al. The species Severe acute respiratory syndrome-related 
coronavirus: classifying $2019-\mathrm{nCoV}$ and naming it SARS-CoV-2. Nat Microbiol. 2020;5:536-44.

2. Koonin EV, Dolja VV, Krupovic M, Varsani A, Wolf YI, Yutin N, et al. Create a megataxonomic framework, filling all principal taxonomic ranks, for realm Riboviria. ICTV. 2019;006G:1-14.

3. World Organization Health (WHO). Statement regardings cluster of pneumonia cases in Wuhan, China. [Internet]. Geneva, Switzerland; 2020. [updated 2020 January 09; cited 2020 Aug 13]. Available from: https://www.who.int/china/news/detail/09-01-2020-|who-statementregarding-cluster-of-neumonia-cases-in-wuhan-china.

4. Li Q, Guan X, Wu P, Wang X, Zhou L, Tong Y, et al. Early transmission dynamics in Wuhan, China, of novel coronavirus-infected pneumonia. N Engl J Med. 2020;382(13):1199-207.

5. Villela DAM. The value of mitigating epidemic peaks of COVID-19 for more effective public health responses. Rev Soc Bras Med Trop. 2020;53:e20200135.

6. Instituto Brasileiro de Geografia e Estatística (IBGE). Estimativas de População dos municípios para 2018. [Internet]. Brasília, Brazil; 2018. [updated 2018 August 29; cited 2020 Aug 13]. Available from: https:// agenciadenoticias.ibge.gov.br/agencia-sala-de-imprensa/2013-agenciade-noticias/releases/22374-ibge-divulga-as-estimativas-de-populacaodos-municipios-para-2018.

7. Ferretti L, Wymant C, Kendall M, Zhao L, Nurtay A, Abeler-Dorner L, et al. Quantifying SARS-CoV-2 transmission suggests epidemic control with digital contact tracing. Science. 2020;368(6491):eabb6936.

8. Sameni R. Mathematical modeling of epidemic diseases; a case study of the COVID-19 coronavirus. arXiv preprint. 2020;arXiv:2003.11371.

9. Panovska-Griffiths J. Can mathematical modelling solve the current Covid-19 crisis?. BMC Public Health. 2020;20:551.

10. Wu JT, Leung K, Leunge GM. Nowcasting and forecasting the potential domestic and international spread of the $2019-\mathrm{nCoV}$ outbreak originating in Wuhan, China: a modelling study. Lancet. 2020;395:689-97.

11. Peak CM, Kahn R, Grad YH, Childs LM, Li R, Lipsitch M, et al. Individual quarantine versus active monitoring of contacts for the mitigation of COVID-19: a modelling study. Lancet Infect Dis. 2020;3099(20)30361-3.

12. Yang C, Wang J. A mathematical model for the novel coronavirus epidemic in Wuhan, China. Math Biosci Eng. 2020;17(3):2708-24.

13. Driessche PVD, Watmough J. Reproduction numbers and sub-threshold endemic equilibria for compartmental models of disease transmission. Math Biosci. 2002;180:29-48.

14. Cidade de São Paulo Saúde. Boletins COVID-19. [Internet]. São Paulo, Brazil; 2020. [updated 2020 August 12; cited 2020 Aug 13]. Available from: https://www.prefeitura.sp.gov.br/cidade/secretarias/ saude/vigilancia_em_saude/doencas_e_agravos/coronavirus/index. php? $\mathrm{p}=295572$.

15. Spencer JA, Shutt DP, Moser SK, Clegg H, Wearing HJ, Mukundan H, et al. Epidemiological parameter review and comparative dynamics of influenza, respiratory syncytial virus, rhinovirus, human coronavirus, and adenovirus. medRxiv. 2020;medRxiv2020.02.04.20020404.

16. Kampf G, Todt D, Pfaender P, Steinmann E. Persistence of coronavirus on inanimate surfaces and its inactivation with biocidal agents. J Hosp Infect. 2020;104:246-51.
17. Tang B, Wang X, Li Q, Bragazzi NL, Tang S, Xiao Y, et al. Estimation of the transmission risk of 2019-nCoV and its implication for public health interventions. J Clin Med. 2020;9(2):462.

18. Haario H, Laine M, Mira A, Saksman E. Dram: Efficient adaptive MCMC. Stat Comput. 2020;16:339-54.

19. Gamerman D, Lopes HF. Markov chain monte carlo: stochastic simulation for bayesian inference. 2nd ed. New York: Taylor and Francis Group; 2006. 342 p.

20. Yang C, Wang J. A cholera transmission model incorporating the impact of medical resources. Math Biosci Eng. 2019;16:5226-46.

21. Cruz PA, Crema-Cruz LC. Mathematical Modeling and Epidemic Prediction of COVID-19 of the State of São Paulo, Brazil. IJAERS. 2020;7:338-47.

22. Fundação Sistema Estadual de Análise de Dados (SEADE). Imprensa. [Internet]. São Paulo, Brazil; 2020. [updated 2020 August 12; cited 2020 Aug 13]. Available from: https://www.seade.gov.br/2020/.

23. Geller C, Varbanov M, Duval RE. Human coronaviruses: Insights into environmental resistance and its influence on the development of new antiseptic strategies. Viruses. 2012;4:3044-68.

24. Abrahão JS, Sacchetto L, Rezende IM, Rodrigues RAL, Crispim APC, Moura C, et al. Detection of SARS-CoV-2 RNA on public surfaces in a densely populated urban area of Brazil: A potential tool for monitoring the circulation of infected patients. Sci Total Environ. 2020:142645.

25. Centers for Disease Control and Prevention (CDC). How COVID-19 Spreads. [Internet]. Atlanta, GA, U.S.; 2020. [updated 2020 September 21; cited 2020 Sep 25]. Available from: https://www.cdc.gov/ coronavirus/2019-ncov/prevent-getting-sick/how-covid-spreads.html.

26. Böhmer MM, Buchholz U, Corman VM, Hock M, Katz K, Marosevic DV, et al. Investigation of a COVID-19 outbreak in Germany resulting from a single travel-associated primary case: a case series. Lancet Infect Dis. 2020;20(8):920-28.

27. Kucirka LM, Lauer SA, Laeyendecker O, Boon D, Lessler J. Variation in False-Negative Rate of Reverse Transcriptase Polymerase Chain Reaction-Based SARS-CoV-2 Tests by Time Since Exposure. Ann Intern Med. 2020;173:4:262-7.

28. He J, Guo Y, Mao R, Zhang J. Proportion of asymptomatic coronavirus disease 2019: A systematic review and meta-analysis. J Med Virol. 2020;10.1002/jmv.26326.

29. Viceconte G, Petrosillo N. COVID-19 R0: Magic number or conundrum?. Infect Dis Rep. 2020;12(1):8516.

30. Linka K, Peirlinck M, Kuhl E. The reproduction number of COVID-19 and its correlation with public health interventions. Comput Mech. 2020;66:1035-1050.

31. Dietz K. The estimation of the basic reproduction number for infectious diseases. Stat Meth Med Res. 1993;2:23-41.

32. Chen TM, Rui J, Wang QP, Zhao ZY, Cui JA, Yin L. A mathematical model for simulating the phase-based transmissibility of a novel coronavirus. Infect Dis Poverty. 2020;9(1):24.

33. Wu J, Tang B, Bragazzi NL, Nah K, McCarthy Z. Quantifying the role of social distancing, personal protection and case detection in mitigating COVID-19 outbreak in Ontario, Canada. J Math Ind. 2020;10(1):15. 